\title{
PERSONALITY, STRESS, AND VENOUS FLOW RATES
}

\author{
Albert J. Silverman* and W. Edward McGough $\dagger$
}

(Received 29 December 1970)

OUR INITIAL interest in the rate of blood flow in superficial limb veins stemmed from observed radiographic differences in speed of travel of intravenously injected radio opaque substances, and from peripheral vascular differences found in Field Dependent (FD) and Field Independent (FI) Ss [1, 2]. Thus, the question was formulated: To what extent were differences in peripheral venous blood flow rates contributing to differences in time of delivery of chemical stimuli to the general circulation? Since the veins are innervated by sympathetic fibers and are responsive to psychological stimuli, a second major interest was in exploring the extent to which venous blood flow rate (in a large superficial arm vein) was modified by psychological stresses, and related to differing personality characteristics.

While vascular dynamics have been studied for many years, to our knowledge the direct measure of rate of blood flow in a superficial limb vein has not been examined from a psychological point of view, if at all.

Venous occlusion plethysmography introduced by Brodie in 1905 [3] remains the standard method of determining forearm blood flow [4]. Although it is certainly altered in response to psychological stresses [4], it does not give a direct and accurate picture of rate of flow in the superficial large forearm veins. Other techniques separate skin from muscle flow [4], but again do not reflect superficial venous blood flow rate. Intravenous injection of radio opaque material followed by cineradiography might be effective but was rejected by us because of the high specific gravity of radio opaque fluids. The ultrasonic blood velocity detector based on the Doppler effect was not available to us [5]. Radioactive tagged substances could be used and the speed of their flow through the arm measured by a counter; however, the use of radioactive substances in normal young subjects might lead to difficulties in obtaining parental consent. We therefore decided to develop another direct technique which would measure venous flow rates, utilizing the standard equipment found in psychophysiology laboratories.

This technique in summary, is as follows: A hydrogen sensitive (platinum oxide tipped) electrode on a teflon probe is threaded into an upper arm vein via an antecubital vein, and a saline I.V. drip is started on the back of the hand in a tributary of that vein. Sodum ascorbate $\left(0.15 \mathrm{~cm}^{3}\right)$ introduced into the hand vein, travels up the arm and causes a voltage change at the electrode in the upper arm vein. (Since it takes only a few molecules to register a change at the electrode, there is no build up period necessary, and therefore no "lag".) The circuit is completed by an electrode pasted to the skin over the point where the platinum electrode lies in the upper arm vein. The voltage change is recorded on a Grass P VII Polygraph employing the P 1-A Grass low level D.C. preamplifier. The Venous Flow Rate (VFR) is then calculated using the distance from injection site on the back of the hand to the platinum oxide electrode, and the time from injection to voltage change.

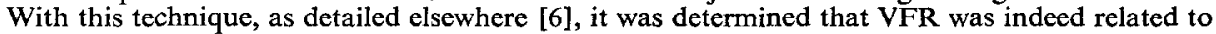
speed of drug action and accounted for about 30 per cent of the variance.

The present paper will report the response of VFR of two stressors, Epinephrine and mental arithmetic, and will explore VFR differences at rest and to the above stresses, among $S$ s with differing personality characteristics. The specific personality variables to be so examined are Anxiety (Taylor

* Department of Psychiatry, University of Michigan Medical Center, Ann Arbor, Michigan.

$\uparrow$ Department of Psychiatry, Rutgers Medical School, New Brunswick, New Jersey. 
A Scale of MMPI); Ego Strength (Barron E.S. Scale on MMPI), and Field Dependency/Independency, employing the data from the experiment noted above [6].

\section{Experimental design}

A random sample of 200 male college students was given a perceptual test battery including the Rod and Frame Test, and other psychological tests including the MMPI. From this group $41 \mathrm{Ss}$ (age 18-21) were selected whose perceptual style differed as follows: 12 FI $S$ s (R \& F) $0.5^{\circ}-1 \cdot 9^{\circ}$ ); 17 "middles", (R \& F $\left.2.0^{\circ}-5.9^{\circ}\right) ; 12 \mathrm{FD} S \mathrm{~s}$, (R \& F $6.0^{\circ}$ or more). Using the Barron E.S. Scale from the MMPI to assess these $S$ s, the mean E.S. value was found to be 48.6 and ranged from 26-59. $11 \mathrm{Ss}$ were culled from this group with E.S. values over 52 (high E.S.), and 11 with values under 47 (low E.S.). Taylor A scores from the MMPI tests administered to the experimental population ranged from 1 to 41 , with the mean T.A. score at 15.6. Ten so called high T.A. Ss were obtained with scores over 20; and ten so called low T.A. Ss were obtained with scores of under 11.

The mean body weights and age of the several segments of these three personality dimensions were essentially identical.

All $S$ s were accustomed to the sound attenuated chamber and to the investigating team, having previously participated in similar psychophysiological experiments. The procedure was explained to the $S \mathrm{~s}$ so as to keep uncertainty at a minimum, and the following experiment performed in a doublyblind manner. $S$ reclined on a hospital bed, the left arm extended through a screen and resting on a table. Quiet music was played through fitted earphones. The vein was then prepared for VFR determination using local anesthesia, and the leads connected to the Grass P VII Polygraph outside the chamber.

The first VFR was determined after a $5 \mathrm{~min}$ rest period, and at $4 \mathrm{~min}$ intervals thereafter. For the 5 th determination, $0.05 \mathrm{mg}$ of Epinephrine was added to the ascorbate, and 5 more VFR were performed. $21 S \mathrm{~s}$ completed this experiment.

An additional $20 S_{s}$ were processed through the above procedure, and were then presented with brief verbal instructions alerting them to expect an arithmetic problem. After the next VFR, a moderately difficult, but solvable arithmetic problem was presented and two more VFR's were obtained, following which the experiment was terminated. Thus a total of $41 \mathrm{Ss}$ were processed through rest and Epinephrine, and $20 \mathrm{Ss}$ through arithmetic.

Statistical analyses including analyses of variance (two factor type with repeated measures), and $t$ tests, compared the extremes of each personality dimension. Middle $S$ s were identified but not statistically examined.

\section{Predictions}

Since Epinephrine has, in other studies, led to increases in forearm blood flow, it was predicted that the VFR response to Epinephrine would also be an increase in speed of flow.

Since arithmetic problems have also led to forearm blood flow increases, it was predicted that the response to arithmetic would likewise be an increase in rate, albeit not as great as with Epinephrine.

One of the observations we made in a recent experiment [1], was that it was much more difficult to withdraw blood from the veins of Field Dependent Ss. Presuming that this might be due to excessive vasoconstriction, the prediction was made that FD Ss would reveal a slower VFR in the anticipatory state of the control period, and in response to Epinephrine and arithmetic.

High A. Ss were predicted to show more of a response to the two stressors as were high E.S. Ss.

\section{RESULTS}

\section{$V F R$ response to epinephrine and mental arithmetic}

Figure 1 depicts the mean of four control levels of VFR at about $2.3 \mathrm{~cm} / \mathrm{sec}$. (Rates ranged from $0.5 \mathrm{~cm} / \mathrm{sec}$ to $7.7 \mathrm{~cm} / \mathrm{sec}$.) None of the four determinations was significantly different, one from another. Epinephrine was injected during the 5th VFR determination and this led to a sharp increase in the speed of blood flow through the vein in several successive VFR's, followed by recovery to control levels by the 9th VFR determination. Two further control VFR's were then obtained and showed recovery to the original resting levels.

In contrast, the announcement to anticipate an arithmetic problem as well as the performance of the problem itself, led to a brisk decrease in the rate of blood flow through the vein (i.e. opposite to our prediction).

The increase in VFR in response to Epinephrine was statistically significant $(t=2.51$, d.f. $=24$, $p<0.01$, one-tailed). The decrease in VFR in response to the anticipation of, as well as in association with the arithmetic problem itself, were also significant. (Anticipation: $t=3 \cdot 33, d . f .=19, p<0.01$, two-tailed; arithmetic: $t=3.72, d . f .=19, p<0.01$, two-tailed.) 


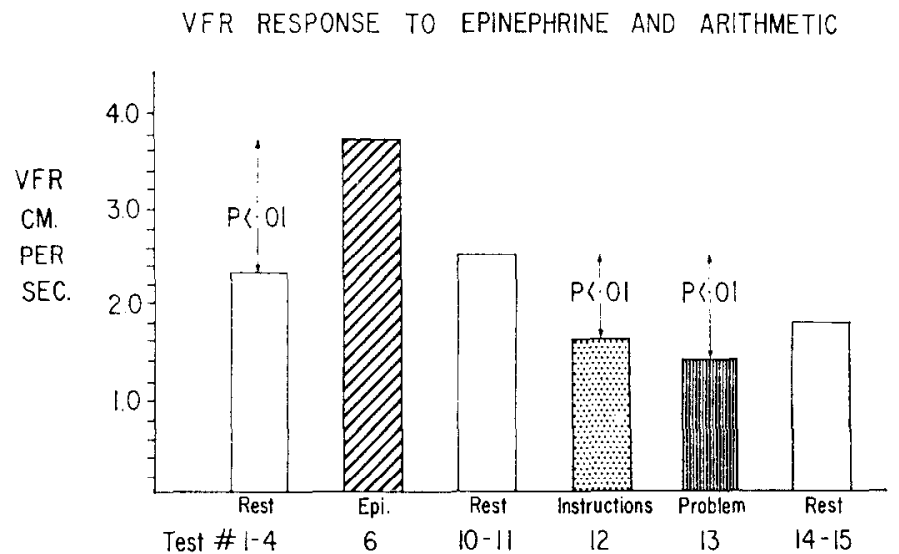

FIG. 1.-Epinephrine $(n=32)$ causes a significant rise, and the instructions for as well as the arithmetic itself, $(n=20)$ causes a significant fall of VFR from resting levels.

\section{VFR differences and personality characteristics}

Chronic anxiety. Our first test to assess VFR differences with personality measures employed the MMPI utilizing the scores which purport to tap chronic anxiety tendencies. These findings are illustrated at the bottom of Fig. 2.

No differences were noted during the control period. The response to Epinephrine was however, quite different in the two groups; the high anxiety $S$ s showing a sharp increase in rate and the low anxiety $S$ s, if anything, a decrease. The analysis of Variance confirms that through time, these two groups respond differently $(F=3.12$, d.f. 4 and $40: p<0.05)$. Prior to Epinephrine the groups are indistinguishable, while immediately after Epinephrine, the VFR of the high anxiety group are significantly higher than the low anxiety group. $(t=2 \cdot 21, d . f .=18, p<0.05$, one tailed. $)$

In response to arithmetic, the Analysis of Variance supports a different response of the two groups through time $(F=2 \cdot 58, P<0.05)$. Inspection also reveals lack of recovery in the high A group, with recovery seen in the low A group. Looking at change scores from pre to post arithmetic, the $t$ test tends to support this differential recovery in the two groups, albeit rather weakly $(t=1.85, d . f .=9$, $p<0 \cdot 1$ ).

Ss scoring in the middle range of this dimension revealed intermediate responses at rest and after Epinephrine, but less of a decrease after arithmetic.

In summary then, high $\mathrm{A} S$ s show a greater response to Epinephrine and a trend to slower recovery from the decreased rate noted with arithmetic, thus partially supporting the predictions.

Ego strength. Roessler et al. [7], have shown that psychophysiologic responses to standard stimuli are more brisk and larger among high than among low ego strength $\mathrm{Ss}$.

VFR findings in our high and low E.S. groups may be seen in the middle section of Fig. 2. Once again middle E.S. Ss revealed intermediate responses. The VFR of the high E.S. group appears consistently higher than the low E.S. group throughout the three stimulus conditions, and indeed the Analysis of Variance suggests a significant E.S. effect during the control period $(F=3 \cdot 86, d . f .1,20$, $0.05<p<0.1)$ and during the Epinephrine period $(F=6.95, d . f .1$ and $10,0.01<p<0.05)$, but not during the arithmetic period.

Although the Analysis of Variance does not support different profiles of response to arithmetic, inspection suggests partial recovery, post arithmetic in high E.S. Ss, but even further decreases in VFR post arithmetic in low E.S. Ss. This suggestion should be followed up in further studies.

In summary then, high E.S. Ss revealed faster venous flow during control and Epinephrine periods, thus providing only partial support of the predictions.

Field dependency. The VFR of Field Dependent and Field Independent $S$ s may be seen at the top of Fig. 2. During the first four control determinations $\Gamma I S$ s appear to rise slightly while $\Gamma D S s$ seem to decline. These changes are in the predicted direction, and a $t$ test performed between the mean changes of the two groups, from the first to fourth VFR, suggests that the groups are acting differently $(t=1.90$, d.f. $=22, p<0.05$, one-tailed $)$.

The response to Epinephrine is also significantly different in the two groups; FI Ss sustain a more prolonged elevation of VFR than do FD Ss. At test Nos. 7 and 8, after Epinephrine, $t$ tests reveal that FI Ss' VFR's are significantly faster than those of FD $S$ s. (At No. 7, $t=2 \cdot 87$, d.f. $=16$, $p=<0.01$, one-tailed; at No. $8, t=1 \cdot 86, d . f .=16, p<0.05$, one-tailed.) 


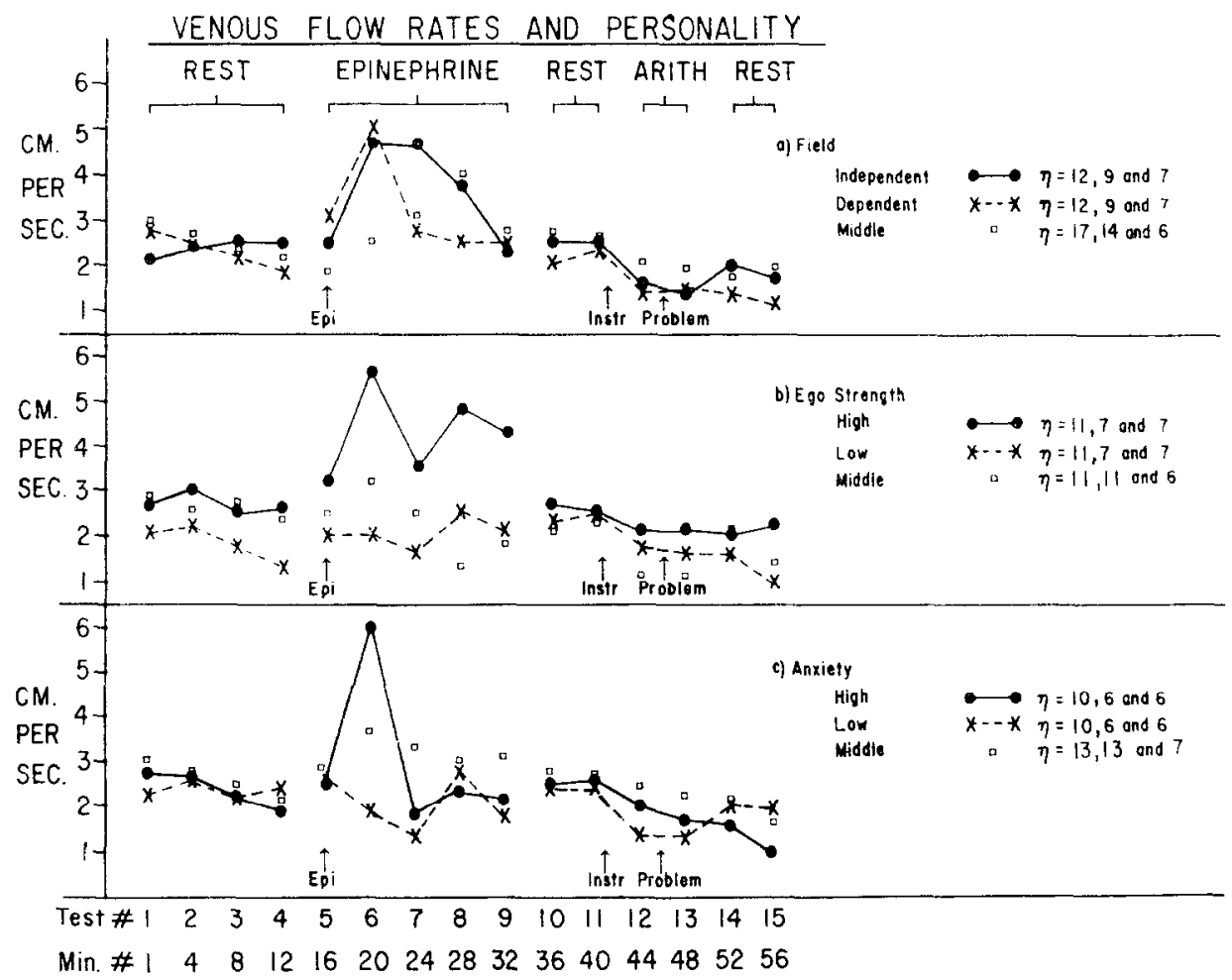

FIg. 2.-Of $41 S$ s completing the first four control tests, 32 completed the Epinephrine sequence and $20 \mathrm{Ss}$ the arithmetic portion. These break down further as shown on the figure. For each subgroup the $n$ 's are given for the three successive portions of the experiment. Note that only 33 of the $S$ s took the MMPI. Nine of the original $41 S$ s were dropped during Epinephrine due to technical difficulties.

Inspection of the response to arithmetic reveals the previously noted significant decrease in both extreme perceptual groups, and FD $S$ s appear to remain low while FI $S$ s speed up somewhat after the problem has been solved. This apparent differential recovery is not, however, supported by statistical tests.

Middle $S$ s are intermediate at rest, show delayed elevation to Epinephrine and less of a decrease in response to arithmetic.

In summary then, FI and FD $S$ s appear to respond differently during the rest period, with FI $S$ s increasing and FD $S$ s decreasing in their rates of superficial venous flow. After Epinephrine, FI $S$ s speed up for a longer period than FD $S$ s. However, no differential response to arithmetic was found The data is thus partially supportive of the predictions made.

Interrelationships between the three personality variables. Plotting the three personality dimensions against one another, we find no significant correlations.

However, there are some interesting trends when T.A. and E.S. are bisected into upper and lower halves. For example, of the 10 FI Ss (on whom we have MMPI data) 7 have T.A. scores below the mean, 3 above. Nine of the 10 FI Ss have E.S. scores above the mean, and only one below. This skewing toward low anxiety and high ego strength, is not secn when cither middle or $\Gamma D S s$ are $c x-$ amined. Both of these latter groups contain roughly equal numbers of high and low $\mathrm{A}$. and high and low E.S. (Table 1).

However, FI-FD differences cannot be accounted for on the above noted differential T.A. and E.S. scores, as can be seen when these latter scores are held constant. 5 FI and 5 FD $S$ s were found to have middle E.S. and middle T.A. scores. When the VFR of these $S$ s were inspected, the perceptual group differences appeared magnified. For example, all 5 FI Ss show progressive increases in VFR over the 4 control determinations, while all 5 FD $S$ s reveal decreases during the same time period. 
(Mean FI $\Delta 1 \mathrm{st}-4$ th $=+1 \mathrm{~cm} / \mathrm{sec} ;$ Mean FD $\Delta 1 \mathrm{st}-4 \mathrm{th}=-2 \cdot 3 \mathrm{~cm} / \mathrm{sec}$.) These findings are significant at a higher level of confidence than obtained in the original $\mathrm{FI} / \mathrm{FD}$ groups $(t=3.00, d . f .=4$, $p=<0.025$, one-tailed).

TABLE 1.-ALthough $41 \mathrm{SS}$ Were SELECTED FOR THE EXPERIMENT ONLY 33 TOOK THE MMPI, THUS ACCOUNTING FOR THE REDUCED NUMBER IN COMPARING THE THREE VARIABLES. FI $S S$ CONTAIN MORE LOW ANXILTY AND HIGH EGO STRENGTH $S$ S THAN EITHER MIDDLES OR FD $S$ s

\begin{tabular}{|c|c|c|c|c|}
\hline \multirow[t]{2}{*}{ Percept. } & \multicolumn{2}{|c|}{ T.A. } & \multicolumn{2}{|c|}{ E.S. } \\
\hline & Lo & $\mathrm{Hi}$ & Lo & $\mathrm{Hi}$ \\
\hline 9 FD & 4 & 5 & 2 & 3 \\
\hline \multirow{2}{*}{$14 \mathrm{M}$} & 7 & 6 & 5 & 1 \\
\hline & 8 & & 3 & 5 \\
\hline \multirow{2}{*}{$10 \mathrm{FI}$} & & 3 & 1 & 2 \\
\hline & 7 & & 0 & 7 \\
\hline
\end{tabular}

\section{DISCUSSION}

The differential VFR with Epinephrine and arithmetic (i.e. the increased speed in response to Epinephrine and the decreased speed in response to an arithmetic problem) was an unexpected but very interesting finding. Previous work with forearm blood flow has shown increases in response both to Epinephrine and a variety of psychological "stresses", including mental arithmetic [4].

In this connection, it should be pointed out that the arithmetic problems which have caused these increases in forearm blood flow (as measured by venous occlusion plethysmography) have usually been difficult problems requiring a great deal of concentration. The problems we posed for our subjects were in contrast, quite possible to answer, were not conceptualized either by the investigators or the $S \mathrm{~s}$ as a "stressor", but merely as alerting mental work.

One explanation of the differential findings with Epinephrine and arithmetic in our experiment might therefore be as follows: Epinephrine, a neurohumor which is associated with action and muscular work leads to an increased speed of flow to facilitate the "fight or flight" reaction. On the other hand, a relatively easy arithmetic problem is merely associated with mental effort. Here, there is no action implication, no threat, no stress and by inference, little production of endogenous Epinephrine. Under these circumstances the limb flow decreases in speed, since there is no need for increased muscle action. If this is correct, more difficult (i.e. more stressful) arithmetic problems should be associated with increased speed of VFR, just as they have caused increased forearm blood flow in previous investigations.

However, it should be pointed out that the mechanisms underlying increases and decreases of VFR (whether constriction or dilatation- neurogenically or/and neurohormonally induced) cannot be inferred from the VFR alone. Clearly, VFR can either increase or decrease in response to constriction, i.e. moderate constriction might increase the rate of flow, while extensive constriction could shut it off. (Hence, the decreases seen with arithmetic could be due to increased constriction.) Dilatation could lead to a slowing of the rate if the muscle vascular bed opened up, but the same amount of dilatation could be associated with a speed up, if blood input to the limb were increased and the muscle vasculature remained constant. Thus, the flow in a 
superficial vein does not necessarily reflect total forearm blood flow. This suggests the desirability of repeating VFR with concomitant venous occlusion plethysmography and other vascular measures to elucidate the mechanisms involved.

Data presently available [4] does not relate forearm blood flow to speed of flow in the large superficial arm vein. Many dynamic factors should be considered in under standing the mechanisms underlying VFR changes. The vessels of different sizes and different parts of the limb are responsive to different stimuli. For example, the small skin vessels play a role in temperature regulation, reflex control being provided by sympathetic vasoconstrictor fibers to the distal small vessels; and to more proximal skin vessels, by sympathetic fibers to the sweat glands, releasing the vasoactive polypeptide bradykinin. Emotional stress or baroreceptor activity do not activate these fibers which respond only to temperature alteration. Changes in flow as a result of temperature shift are confined to the skin vessels, digital vessels and hand vessels. The muscle vessels do not participate.

Blood vessels in human skeletal muscle are supplied with sympathetic vasoconstrictor and vasodilator fibers. Constrictor activity is modified by the amount of stimulation of receptors in thoracic low-pressure vessels. The center controlling the cholinergic vasodilator fiber is activated by emotional stress. A humoral mechanism also exists, Epinephrine probably also playing a role in psychological muscle dilatation in response to emotional stress.

The large superficial forearm veins are innervated with sympathetic constrictor fibers which are reflexly responsive to a variety of central influences including emotional stimuli. For example, the isolated venous segment reveals increases in pressure to emotional and painful stimuli [8]. A humoral mechanism probably also exists. Norepinephrine and Epinephrine introduced into the isolated venous segment also cause pronounced rises in pressure. (Thus in our experiment it seems likely that the increased VFR with the introduction of Epinephrine into the vein, was due to constriction.)

The digital vessels are also innervated with sympathetic constrictor fibers, responsive to temperature changes as well as to emotional stimuli. The state of affairs in the hand seems less clear, heat stimuli causing increases in hand flow, and emotional stimuli having variable results.

The vascular dynamics underlying alterations in rate of blood flow in the forearm superficial vein might thus better be elucidated by experiments employing quantitatively and qualitatively different psychological stressors and stimuli, with simultaneous measures of digital flow (photoelectric plethysmography), forearm blood flow (venous occlusion plethysmography) and venous flow rate determinations. Determinations of venous pressures in the isolated venous segment should also be included, but since it is not feasible to include all the above measures simultaneously, various combinations would be employed. Clearly both limbs must be used and the assumption made that in general the left and right limbs operate in synchrony.

It should also be recalled that in this experiment, the arithmetic problem followed the administration of I.V. Epinephrine through the same vein used to measure the VFRs. Epinephrine as noted previously, has a direct vasoconstrictive effect on the veins when its concentration is relatively high. This constriction was sometimes apparent as transient blanching around the site of injection. Although transient, it is possible that this may have affected the VFRs in the mental arithmetic part of the 
experiment. VFR experiments are therefore planned to determine the effect of mental arithmetic alone on the VFR, without the possible "contamination" associated with previously injected vasoconstrictive substances.

For the time being, it may be of interest in and of itself that speed of blood flow in a large superficial arm vein in response to two different external "stressors" does not appear to be unidirectional.

Of equal interest was the demonstration that familiar personality dichotomies also reveal differences in VFR. The gradual decrease in speed among FD $S$ s at rest, in contrast with an increase in speed among FI $S$ s, may be related to findings, which we had speculated to be due to higher resting levels of arousal in FD Ss. We had previously noted (1) difficulty in drawing blood from FD $S \mathrm{~s}$; and in a recent experiment, as yet unpublished, determined that in resting conditions the veins of FD Ss gradually show greater pressure than FI $S$ s. This suggests that we may later discover the blunted speed of flow in response to Epinephrine to be associated with excessive constriction in the FD group.

The choice of "Ego Strength" as a second variable was determined by Roessler's findings of more "brisk" responses among high E.S. Ss. It should be pointed out that the spread of E.S. values in Roessler's population was much broader than in our $S$ s, all of whom were clinically healthy and whose E.S. values were in general higher. In spite of this, the "low" E.S. Ss (E.S. values 26-47) showed consistently slower VFR than the "high" E.S. Ss (E.S. values 52-59) and again suggests the greater capacity of high E.S. Ss to respond.

The increase in speed of flow in response to Epinephrine among high $\mathrm{A}$ as opposed to low A Ss reflects the lability of this group, self selected in part by their admission of strong psychophysiological reactions to stress.

Many other investigations have demonstrated that personality measurements like Barron's Ego Strength and Taylor Anxiety scores are associated with physiological differences and their inclusion in this presentation was essentially to test whether VFR would also reveal these differences.

The fact that the low E.S. and high A Ss showed some evidence of slowing after the arithmetic problem is ovcr, is intercsting in itsclf and should be explored further.

Of similar interest is the relationship between the three personality variables.

Correlations performed between the three personality variables revealed no significant relationships, but a weak tread was identified toward a skewing of low anxiety and higher Ego Strength among FI $S$ s. When the perceptual groups were equated for these two variables, the perceptual group differences were more impressive, suggesting that independent of other personality factors, Field Dependency per se is a differentiating variable in psychophysiological responses.

Thus, as usual, this experiment, while in general supporting our several predictions moderately well, has also uncovered unexpected and striking apparent differences in rate of flow in response to two external stressors and raised many important questions, once again underscoring the complexity of psychophysiological mechanisms in man.

\section{SUMMARY}

Previously noted vascular differences between Field Dependent (FD) and Field Independent (FI) $S$ s led to an interest in Venous Flow Rates (VFR) in these two groups. Blood flow rate in a large arm vein was determined by threading a hydrogen 
sensitive (platinum-oxide tipped) electrode into an upper arm vein, and introducing Sodium Ascorbate (as a source of $\mathrm{H}^{+}$) into a tributory on the back of the hand. VFR was measured by dividing the time for $\mathrm{H}^{+}$to reach the electrode, into the distance traveled.

With this technique, 4 resting VFR in $41 S \mathrm{~s}$ were found to average $2 \cdot 3 \mathrm{~cm} / \mathrm{sec}$.

Epinephrine caused a significant increase in rate of flow, while the instructions to anticipate an oral arithmetic problem, and the problem itself, caused a significant decrease.

FI $S$ s rose and FD $S$ s fell during the four resting VFR. FI $S$ s also revealed more persistent elevation in VFR than FD $S$ s in response to Epinephrine.

High Ego Strength Ss (Barron Scale of MMPI) had faster rates at rest and after Epinephrine, than low E.S. Ss.

High Anxiety $S$ s (Taylor Scale of MMPI) revealed faster rates during Epinephrine and less recovery from arithmetic than low A Ss.

Acknowledgement-This investigation was supported by PHS Research Grant MH 08804 from the National Institute of Mental Health.

\section{REFERENCES}

1. McGough W. E., Silverman A. J. and Bogdonoff M. D. Patterns of fat mobilization in field dependent and field independent subjects. Psychosom. Med. 27, 345 (1965).

2. Silverman A. J. et al. Perceptual correlates of the physiological response to insulin, Psychosom. Med. 29, 252 (1967).

3. Brodie T. G. and Russell A. E. On the determination of the rate of blood flow through an organ. J. Physiol. 32, XLVII (1905).

4. SHEPHERd J. T. Physiology of the circulation in human limbs. In Health and Disease. Sauders, Philadelphia (1963).

5. SUMNER D. F., BaKer D. W. and Strandness D. E. JR. The ultrasonic velocity detector in a clinical study of venous disease. Arch. of Surgery 97, 75 (1968).

6. MCGough W. E., Silverman A. J. and LeE B. Venous flow rate and its effect on the delivery of an intravenous stimulus in psychophysiological studies. J. Psychosom. Res. 13, 167 (1969).

7. ROESSLER R., ALEXANDER A. and GREENFIELD V. Ego strength and physiological responsivity. Arch. Gen. Psychiat. VIII, 142 (1963).

8. Cohen S. I., Bondurant S. and Silverman A. J., Psychophysiological influences on peripheral venous tone. Psychosom. Med. 22, 106 (1960).

Abstract-A technique of directly measuring Venous Flow Rate (VFR) in a large superficial limb vein is described.

With this technique, increases in VFR were noted in response to Epinephrine; and decreases in VFR, in response to the anticipation of, and the performance of an alerting mental arithmetic problem.

High Ego Strength $S s$ revealed higher rates of flow than low E.S. Ss at rest and after Epinephrine.

High Anxiety $S$ s revealed more of an increase of rate of flow in response to Epinephrine and a less brisk recovery after arithmetic than low anxiety $S$ s.

During resting conditions Field Independent $S$ s revealed gradual increase in VFR, while Field Dependent $S$ s revealed decreases. These differences were intensified when F.I. and F.D. $S$ s were equated for ego strength and anxiety scores.

F.I. $S$ s also revealed a more prolonged increase in rate of flow in response to Epinephrine than Field Dependent $S$ s. 